\title{
Marchiafava-Bignami disease: A case report
}

\author{
A Ellepola, CA Abayaweera
}

\begin{abstract}
Marchiafava-Bignami disease (MBD) is a rare neurodegenerative disorder affecting the corpus callosum. It is usually associated with chronic use of alcohol leading to nutritional deficiencies. However, its aetiology is not completely understood. Clinical features of this condition include paralysis, confusion, cognitive deficits, aggression, apraxia, ataxia, coma and seizures. Degeneration of the corpus callosum can be identified on MRI scan of the brain. The prognosis of MBD is variable. There are reported cases of
\end{abstract}

complete recovery, partial recovery and death. Administration of high doses of thiamine, vitamin B complexes and steroids has shown benefits in some cases. In this case report, a 48-year old chronic alcohol user was treated for MBD with high doses of thiamine, and made a good recovery.

Key words: Marchiafava-Bignami disease, corpus callosum

SLJ Psychiatry 2019; 10(1): 22-24

\section{Introduction}

Marchiafava-Bignami disease (MBD) is a rare progressive neurodegenerative disorder. It was first described in 1903, by two Italian pathologists, Amico Bignami and Ettore Marchiafava (1). They reported necrosis of corpus callosum associated with the condition (1).

MBD is usually associated with chronic alcohol use and nutritional deficiencies. In the past, a definitive diagnosis of MBD was made only at the autopsy. In current practice, neuroimaging with MRI scans of the brain is an important part of the diagnosis. Although not completely understood, primary demyelination, degeneration and the atrophy of corpus callosum is a characteristic pathology of this disease (2). There have been reported cases of MBD with no history of alcohol consumption as well (3, 4). Deficiency of vitamin B is the most likely contributor for the condition (5). Administration of high doses of thiamine is effective in only a proportion of patients. Others show a poor prognosis. Early detection and management seems to improve the prognosis. Mortality rates are high in patients with poor outcome. Variable outcomes with administration of vitamin B had prompted research into treatment with other medications. Treatment with steroids have shown benefits in few cases (6). An efficacious and effective treatment is yet to be discovered, for this condition with unpredictable outcome.
The presentation of MBD can be acute, subacute or chronic. Paralysis, confusion, aggression, apraxia, ataxia, coma and seizures are the common symptoms of the condition (7).

\section{Case Report}

A 48-year old male clerk presented with confusion, ataxia, dressing apraxia and agitation for a period of one week.

The patient also had a history of daily consumption of alcohol for over 10 years. He consumed up to two bottles of alcohol per day. There was a history suggestive of alcohol withdrawal seizures and delirium tremens in the past. He continued to drink alcohol despite knowing its harm. He had neglected responsibilities at home and at work resulting in a number of inquiries at his work place. He met DSM 5 criteria for the diagnosis of alcohol dependence. There was no history of any other medical or psychiatric co-morbidity. He showed no sign of an infection. His family reported that he refused medical help to treat alcohol dependence in several occasions.

Following presentation to services, the patient was admitted to the psychiatric inpatient unit at Teaching Hospital, Anuradhapura for further management. High doses of parenteral thiamine and a flexible dose of oral chlordiazepoxide were administered. The patient was kept under close observation. 

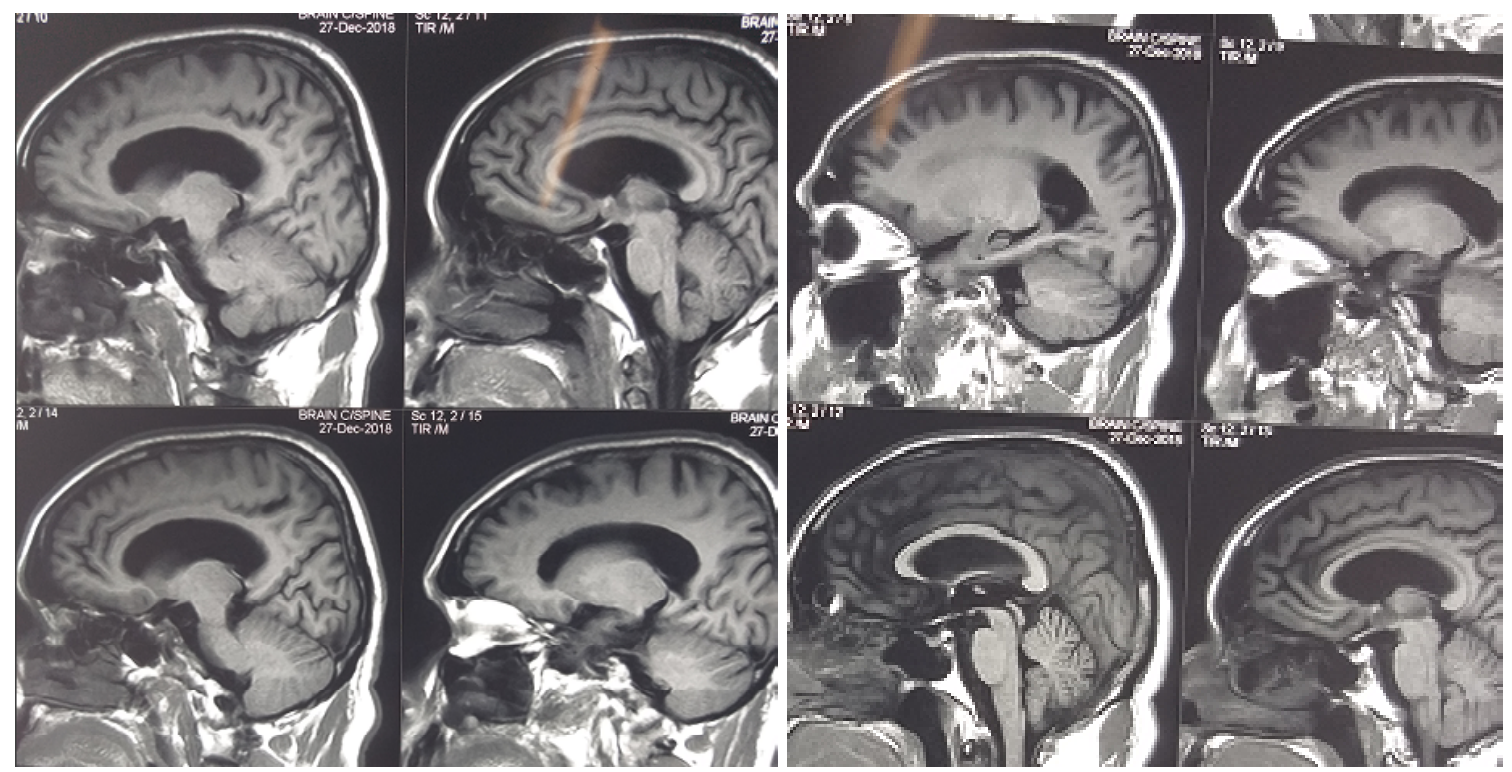

Figure 1. MRI brain showing 'sandwich sign' and hypodensity of the corpus callosum.

Basic blood investigations and radiological investigations were arranged. His liver functions were deranged with markedly elevated aspartate aminotransferase (AST or SGOT) and alanine aminotransferase (ALT or SGPT) and gamma-glutamyl transferase (GGT). He also had a mild anaemia as shown by the full blood count. Early computed axial tomograph (CT) and magnetic resonance imaging (MRI) of brain revealed a hypodensity in corpus callosum. The MRI of brain also showed the 'sandwich sign' where there was an involvement of central layer of corpus callosum with sparing of the rest of it (Figure 1).

The patient's confusion settled first with the treatment. The patient became well oriented in time, place and person. Mini mental state examination (MMSE) and the Montreal Cognitive Assessment (MoCA) showed no deficits. His agitation subsided, and there were no symptoms of alcohol withdrawal by the end of first week of the hospital stay. His ataxia and apraxia showed some improvement after two weeks of treatment. The patient was discharged home after 3 weeks of hospital stay, and he was prescribed disulfiram and oral thamine on discharge. Fortnightly out-patient follow up, motivational interviewing and supportive counselling was arranged. The patient showed a complete recovery on his first outpatient clinic visit. He resumed work and has remained abstinent during the two months following hospital discharge.

\section{Discussion}

There is a wide variation in the clinical presentation of MBD. While a proportion of patients show poor outcome such as coma or death, others may partially or fully recover. Prediction of prognosis may be difficult. The outcome of MBD depends partly on its aetiology, and time taken to initiate treatment. This is further compli- cated by the controversy around its pathophysiology, and treatment (7).

Patients may present acutely with confusion, seizures, coma or death. Neurological and neuropsychiatric presentations such as ataxia, apraxia, dysarthria and dementia may be the presentation in subacute and chronic cases (7). Demonstration of hypodensity in the corpus callosum on CT or MRI brain, with typical clinical features is needed for the diagnosis (8).

The decision regarding a specific treatment is difficult due to the uncertainties of aetiology of this condition. The evidence indicates that thiamine, as well as corticosteroids and vitamin B complexes may be helpful in some patients with a good prognosis. However a proportion of patients fail to recover from the condition, despite early and prompt initiation of treatment. The patient in this case report completely recovered with administration of thiamine. He showed no long-term adverse consequences of the condition following recovery, and remained abstinent from alcohol at two months follow-up after discharge.

\section{Declaration of interest}

None declared

A Ellepola, CA Abayaweera, Teaching Hospital, Anuradhapura

Corresponding author: A Ellepola

Email: anu.ellepola@gmail.com

https://orcid.org/0000-0001-9699-2777 


\section{References}

1. Marchiafava E, Bignami A. Sopra un alterazione del corpo calloso osservata in soggetti alcoolisti. Riv Patol Nerv 1903; 8: 544-9.

2. Hlaihel C, Gonnaud PM, Champin S, Rousset H, TranMinh VA, Cotton F. Diffusion-weighted magnetic resonance imaging in Marchiafava-Bignami disease: followup studies. Neuroradiology 2005; 47(7): 520-4.

3. Celik Y, Temizoz O, Genchellac H, Cakir B, Asil T. A nonalcoholic patient with acute Marchiafava-Bignami disease associated with gynecologic malignancy: paraneoplastic Marchiafava-Bignami disease? Clin Neurol Neurosurg 2007; 109(6): 505-8.

4. Rusche-Skolarus LE, Lucey BP, Vo KD, Snider BJ. Transient encephalopathy in a postoperative non- alcoholic female with Marchiafava-Bignami disease. Clin Neurol Neurosurg 2007; 109(8): 713-5.

5. Helenius J, Tatlisumak T, Soinne L, Valanne L, Kaste M. Marchiafava-Bignami disease: two cases with favourable outcome. Eur J Neurol 2001; 8(3): 269-72.

6. Kikkawa Y, Takaya Y, Niwa N. A case of MarchiafavaBignami disease that responded to high-dose intravenous corticosteroid administration. Rinsho Shinkeigaku 2000; 40(11): 1122-5.

7. S Raina, DM Mahesh, J Mahajan, SS Kaushal, D Gupta, DS Dhiman. Marchiafava Bignami Disease. Journal Assoc Physicians India 2008; 56: 633-5.

8. A Heinrich, U Runge, AV Khaw. Clinicoradiologic subtypes of Marchiafava-Bignami disease. J Neurol 2004; 251(9): 1050-9. 